\title{
CD147 blockade as a potential and novel treatment of graft rejection
}

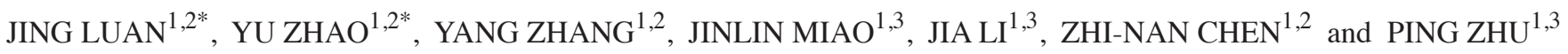 \\ ${ }^{1}$ National Translational Science Center for Molecular Medicine, The Fourth Military Medical University; ${ }^{2}$ Cell \\ Engineering Research Center and Department of Cell Biology, The Fourth Military Medical University; ${ }^{3}$ Department of \\ Clinical Immunology, Xijing Hospital, The Fourth Military Medical University, Xi'an, Shaanxi 710032, P.R. China
}

Received March 16, 2017; Accepted August 1, 2017

DOI: $10.3892 / \mathrm{mmr} .2017 .7201$

\begin{abstract}
Cluster of differentiation (CD)147 is highly involved in the $\mathrm{T}$ cell activation process. High CD147 expression is observed on the surfaces of activated $\mathrm{T}$ cells, particularly CD4+ T cells. In organ transplantation, it is important to prevent graft rejection resulting from the excessive activation of T cells, particularly CD4+ T cells, which exhibit a key role in amplifying the immune response. The present study aimed to investigate the effects of CD147 blockade in vitro and in vivo and used a transplant rejection system to assess the feasibility of utilizing CD147 antibody-based immunosuppressant drugs for the treatment of graft rejection. The effects of CD147 antibodies were evaluated on lymphocyte proliferation stimulated by phytohemagglutinin or CD3/CD28 magnetic beads and in a one-way mixed lymphocyte reaction (MLR) system in vitro. For the in vivo analysis, an allogeneic skin transplantation mouse model was used. CD147 antibodies were effective against lymphocytes, particularly CD4+T lymphocytes, and were additionally effective in the one-way MLR system. In the allogeneic skin transplantation mouse model, the survival of transplanted skin was extended in the CD147 antibody-treated group.
\end{abstract}

Correspondence to: Dr Zhi-Nan Chen or Professor Ping Zhu, National Translational Science Center for Molecular Medicine, The Fourth Military Medical University, 169 Changle West Road, Xi'an, Shaanxi 710032, P.R. China

E-mail: zhinanchen@fmmu.edu.cn

E-mail: zhuping@fmmu.edu.cn

*Contributed equally

Abbreviations: CCK-8, Cell Counting kit 8; FITC, fluorescein isothiocyanate; FK506, tacrolimus; IFN- $\gamma$, interferon $\gamma$; IL-17, interleukin 17; IL-4, interleukin 4; mAb, monoclonal antibody; MHC, major histocompatibility complex; MLR, mixed lymphocyte reaction; MS, multiple sclerosis; PBMCs, peripheral blood mononuclear cells; PE, phycoerythrin; PI, propidium iodide; RA, rheumatoid arthritis

Key words: CD147 antibody, immunosuppressant, graft rejection, treatment
Furthermore, the level of inflammatory cell infiltration in transplanted skin was reduced. CD147 blockade decreased the serum levels of interleukin (IL)-17 and the proportions of peripheral blood CD4+ and CD8+ memory T cells. The data demonstrated that CD147 blockade suppressed skin graft rejection, primarily by suppressing $\mathrm{CD} 4+\mathrm{T}$ and memory $\mathrm{T}$ cell proliferation, indicating that CD147 exhibits great potential as a target of immunosuppressant drugs.

\section{Introduction}

Transplantation remains the best treatment option for correcting certain types of organ failure and tissue damage, e.g., that of the kidneys, heart, and lungs (1). In organ transplantation, it is always a main goal to prevent rejection. However, the long-term use of globally nonspecific immunosuppressant drugs to prevent rejection is associated with serious risks, including a weakened immune system, infection, and cancer, as well as graft loss due to their toxicity $(1,2)$. Thus, identifying safe, target-specific immunosuppressive agents for promoting allograft survival is a high priority.

Acute rejection is clinically the most common and is mainly mediated by cellular immune responses; this type of rejection can occur any time from a week to months after transplantation. $\mathrm{T}$ cells, especially CD4+ T cells from the recipient, play a key role in acute transplantation rejection. As a trigger, CD4+ T cells amplify the downstream signal. By recognizing HLA-II antigens and interacting with them, CD4+ T cells cause antigen-presenting cells to release interleukin (IL)-1, which could promote CD4+ T cells releasing IL-2. IL-2 can, in turn, promote CD4+ T cell proliferation and provide an auxiliary signal for CTL cell differentiation. Furthermore, CD4+ T cells produce IL-4 and IL-5 to promote $B$ cell differentiation and the secretion of antibodies against the graft to participate in the rejection reaction. Different CD4+ $\mathrm{T}$ cell subsets execute different functions during transplant rejection. There have been studies supporting the notion that the balance of the Th1/Th2 cell proportion is a determining factor in this process. Th1 cells mainly participate in graft rejection, especially in acute rejection; in contrast, Th2 cell activation leads to the formation of transplantation tolerance $(3,4)$. Furthermore, cytokines such as Th1-related interferon (IFN)- $\gamma$ and Th2-related IL-2, IL-4 
and IL-10 participate in the development of rejection (5). As research continues, CD4+ T cell subsets, e.g., Th17 and Treg, are considered to be the two main types involved in allograft rejection and survival $(6,7)$.

In addition to the above-mentioned mechanisms of CD4+ T cell subsets, memory T cells (both CD4+ and CD8+) have been found to be involved in development of the rejection reaction (8-10). In a primate model, the elimination of memory $\mathrm{T}$ cells has been shown to induce the body's immune tolerance (11). It has also been reported that infusion of sensitized memory CD4+ T cells produces acute rejection in mice that have undergone abdominal heterotopic heart transplantation. In addition, infusion of sensitized memory CD4+ T cells has been shown to significantly shorten the survival times of transplanted hearts, and this effect cannot be reversed by commonly used immunosuppressants $(12,13)$. Clinical studies have shown that the prognoses of patients who have undergone liver transplantation are dependent on patient CD8+ T memory cell status (14). The results of an in vivo study have shown that endogenous donor-reactive CD8+ memory $\mathrm{T}$ cells infiltrate the transplanted hearts of mice within a few $h$ after reperfusion and secrete IFN- $\gamma$, causing inflammation (15). It is well known that antibody-mediated $\mathrm{T}$ cell depletion is one of the most potent immunosuppressant therapies. This therapy is increasingly used as an induction therapy in organ transplantation (16). However, T cell homeostasis after depletion therapy leads to a predominance of memory $\mathrm{T}$ cells, which are more potent than naïve $\mathrm{T}$ cells in mediating graft rejection and present a major obstacle to achieving tolerance $(17,18)$.

CD147 is a cell-surface glycosylated transmembrane protein that belongs to the immunoglobulin superfamily. This protein serves multiple biological functions and is widely expressed in many tissues and cell types, such as normal brain tissue, tracheal, lung, and breast tissues, as well as lymphocytes and neutrophils (19,20). High CD147 expression is involved in many different diseases. In the immune system, CD147 participates in different stages of $\mathrm{T}$ cell activity, including development, activation, proliferation, migration, and adhesion $(19,21,22)$. It is worth mentioning that CD147 has been identified as a T cell activation-related antigen (M6) expressed in phytohemagglutinin (PHA)-activated T lymphocytes (23). In a previous study at our institute, we found that CD147 participated in immunological synapse formation by co-localizing with CD48 molecules on the surface of T cells. In addition, blocking CD147 decreased intracellular calcium mobilization and affected protein tyrosine phosphorylation upon CD3/TCR stimulation, all of which are very important in the process of $\mathrm{T}$ cell activation. Interestingly, CD147 expression was clearly increased upon $\mathrm{T}$ cell activation, and this trend was the most obvious in CD4+ T cell subsets (24). This information suggests that CD147 blockade is a potential way to inhibit the function of T cells, especially CD4+ T cells.

ABX-CBL, also known as gavilimomab, is a hybridoma-generated murine IgM monoclonal antibody (mAb) against CD147. Studies using ABX-CBL for the treatment of steroid-resistant acute GVHD have shown encouraging results. ABX-CBL did not yield improvements in outcomes compared with anti-thymocyte globulin and therefore did not meet the criteria for FDA approval; however, it did show activity against CD147, which suggests that CD147 is an effective target for the treatment of GVHD (25-27). The effect of CD147 blockade on allograft rejection has never been investigated before. Thus, this study was performed to investigate whether the blockade of CD147 can inhibit the rejection reaction and to determine whether CD147 antibodies could be developed as specific immunosuppressors for the graft rejection response.

\section{Materials and methods}

Antibodies and reagents. Humanized mAbs (5A12) against CD147 were generated and identified in our laboratory. A purified human IgG1 isotype control antibody (Clone: ET901) was purchased from BioLegend, Inc. (San Diego, CA, USA). An anti-mouse CD147 functional-grade purified antibody (Clone: RL73) and its isotype control (Clone: eBR2a) were purchased from eBioscience (San Diego, CA, USA). Tacrolimus (FK506) was purchased from KeHao (Wuhan, Hubei, China). Fluorescein isothiocyanate (FITC)-conjugated mouse anti-human CD4 and phycoerythrin (PE)-conjugated mouse anti-human CD3 antibodies, Foxp3 staining buffer and a cell activation cocktail used for intracellular staining were purchased from BioLegend. PE-Cy7-conjugated rat anti-mouse IFN- $\gamma$, PE-conjugated rat anti-mouse IL-4, Alexa 647-conjugated rat anti-mouse IL-17, FITC-conjugated rat anti-mouse CD4, allophycocynanin-H7-conjugated rat anti-mouse CD8, PE-Cy7-conjugated rat anti-mouse CD44, BUV737-conjugated rat anti-mouse CD62 L, allophycocyanin-conjugated rat anti-mouse CD25, and PE-conjugated rat anti-mouse Foxp3 antibodies were purchased from BD Biosciences (San Diego, CA, USA).

Cell isolation. Human peripheral blood was obtained from healthy volunteers who provided informed consent. In brief, lymphocytes were isolated by density gradient centrifugation over Lymphocyte Separation Medium (MP Biomedicals, LLC, Santa Ana, CA, USA) according to the manufacturer's instructions. CD4+ T cells were isolated by negative selection using a magnetic cell separation system according to the manufacturer's instructions (Miltenyi Biotec, Bergisch Gladbach, Germany). The isolated cells were then washed once and resuspended in phosphate-buffered saline (PBS). After incubation with PE-conjugated mouse anti-human CD3 and FITC-conjugated mouse anti-human CD4 antibodies, the purities were determined by flow cytometry (FACSCalibur; BD Biosciences). The purity of CD3+ CD4+ T cells consistently ranged from $92-95 \%$.

Cell proliferation, apoptosis, and cytokine production. Human lymphocytes and CD4+ T cells were separately plated in $96-$ well plates at a density of $3 \times 10^{5}$ cells/well in $200 \mu 1$ of RPMI 1640 supplemented with $10 \%$ fetal bovine serum. Then, the cells were stimulated with PHA $(2 \mu \mathrm{g} / \mathrm{ml}$; Sigma, St. Louis, MO, USA) or co-cultured with CD3/CD28 magnetic beads (at a bead-to-cell ratio of 1:1; Gibco Life Technologies, Carlsbad, CA, USA) with or without the addition of 5A12 (six wells were performed in parallel in each group). Lymphocyte proliferation was measured using Cell 
Counting kit 8 (CCK-8) assay (Engreen Biosystem, Beijing, China) according to the manufacturer's instructions. Cell apoptosis was evaluated by flow cytometry (FACSCalibur; BD Biosciences) using an FITC Annexin V Apoptosis Detection kit with propidium iodide (PI; BioLegend, Inc.). In brief, cells were washed twice and then resuspended at a concentration of $0.5-1.0 \times 10^{7}$ cells $/ \mathrm{ml}$. Next, $100 \mu \mathrm{l}$ of cell suspension was transferred to each well and incubated with $5 \mu \mathrm{l}$ of FITC Annexin V and $10 \mu \mathrm{l}$ of PI at room temperature for $15 \mathrm{~min}$. The samples were then ready for testing. Cells were collected by flow cytometry (FACSCalibur; BD Biosciences).

One-way mixed lymphocyte reaction $(M L R)$. The experimental method used here has been previously described $(28,29)$. Peripheral blood mononuclear cells (PBMCs), i.e., lymphocytes, from human volunteers were used as responder cells, and splenocytes from C57BL/6 mice were used as stimulator cells. The lymphocytes were suspended in RPMI 1640 supplemented with $10 \%$ fetal bovine serum at a density of $1.5 \times 10^{6}$ cells $/ \mathrm{ml}$ and marked as sample A. Two-thirds of the splenocytes were removed and incubated with mitomycin C (Sigma) at a final concentration of $25 \mu \mathrm{g} / \mathrm{ml}$ in RPMI 1640 (without fetal bovine serum) at $37^{\circ} \mathrm{C}$ for $30 \mathrm{~min}$. The remaining splenocytes were used as a control. After incubation, the splenocytes were washed twice with PBS, resuspended at the same density of $1.5 \times 10^{6}$ cells $/ \mathrm{ml}$ in RPMI 1640 supplemented with $10 \%$ fetal bovine serum and marked as sample B. Samples A and B were suspended and mixed at a ratio of $1: 1$; then, $200 \mu 1$ of the cell suspension was pipetted into round-bottomed 96 -well plates. Cells were cultured with or without $5 \mathrm{~A} 12$ for $96 \mathrm{~h}$ at $37^{\circ} \mathrm{C}$ and $5 \% \mathrm{CO}_{2}$ in a humidified atmosphere. Then, lymphocyte proliferation and cytokine production were detected using human IFN- $\gamma$, IL-4 and IL-17 ELISA kits (Dakewe Bio Engineering Co., Ltd., Shenzhen, China).

Animals. Male C57BL/6 (H-2 $\left.{ }^{\mathrm{b}}\right)$ and BALB/c $\left(\mathrm{H}-2^{\mathrm{d}}\right)$ mice were obtained from Beijing Vital River Laboratory Animal Technology Co., Ltd. (Beijing, China) and housed in a specific pathogen-free facility in microisolator cages at The Fourth Military Medical University laboratory animal center. All animals used were 8-10 weeks old. All protocols were approved by the institutional Ethics Committee on animal use of The Fourth Military Medical University of Science and Technology. The skin allografts were placed using a standard technique $(30,31)$, and operations were performed under aseptic conditions. In brief, mice were euthanized under general anesthesia. Full-thickness skin grafts $1 \mathrm{~cm}$ in diameter were obtained from the tails of C57BL/6 (H-2b) donor mice, transplanted onto the backs of BALB/c $(\mathrm{H}-2 \mathrm{~d})$ recipient mice, and protected by a bandage for 9 days. The transplanted grafts were scored visually daily, and a graft was considered fully rejected when $80 \%$ of the tissue was necrotic. Drugs were administered to the mice every day via intraperitoneal injection (antibodies, $5 \mathrm{mg} / \mathrm{kg}$; FK506, $0.5 \mathrm{mg} / \mathrm{kg}$ ) from the day of the grafting (D0) to the day of rejection. The mice in the autograft and allograft control groups were injected with an equal volume of saline. Mice from each group were randomly selected and euthanized under general anesthesia on D10.
Blood was then collected from the orbital sinus. Plasma was obtained by centrifugation and stored at $-20^{\circ} \mathrm{C}$ for later use. Cytokine production was assessed using a MILLIPLEX MAP kit (EMD Millipore Corporation, Billerica, MA, USA) according to the manufacturer's instructions. Splenocytes and peripheral blood were stained with antibodies according to conventional treatment methods and then immediately assessed using an LSRFortessa X-20 flow cytometer (BD Biosciences). Skin grafts were harvested and fixed in $4 \%$ formalin for histological studies. Histological analysis of the skin grafts was performed by staining 5-mm sections of paraffin-embedded tissues with hematoxylin and eosin. Inflammatory cells were counted by examining 10 different visual fields under a microscope at magnification, $\mathrm{x} 400$ in a blinded manner.

Statistical analysis. The results presented are representative of a minimum of three experiments and are expressed as the mean \pm standard deviation unless otherwise stated. Multiple comparisons were performed with one-way ANOVA. Graft survivals were analyzed by the Kaplan-Meier method using the log-rank test. For analysis of T cell responses with flow cytometry method, nonparametric Mann-Whitney U tests were performed. GraphPad software (GraphPad Software, Inc. La Jolla, CA, USA) was used for the above analyses, and $\mathrm{P}<0.05$ was considered significant.

\section{Results}

Proliferation of $C D 4+T$ cells from human peripheral blood was inhibited by $5 A 12$. We have previously reported that CD147 expression is clearly increased upon T cell activation, especially in CD4+ T cell subsets (24). We first verified the effects of the humanized CD147 mAb 5A12 on the proliferation of $\mathrm{CD} 4+\mathrm{T}$ cells isolated from the peripheral blood of healthy people. When CD4+ T cells were stimulated with PHA or CD3/CD28 magnetic beads, marked proliferation was induced. The addition of 5A12 to the cultures strongly inhibited this proliferation by 28 and $77 \%$ in the PHA- and CD3/CD28-stimulated groups, while the resting lymphocytes were unaffected (Fig. 1A and B). In addition, 5A12 inhibited proliferation in a dose-dependent manner; the minimum significant concentrations for PHA-stimulated and CD3/CD28 magnetic bead-stimulated CD4+ T cells were $100 \mu \mathrm{g} / \mathrm{ml}$ and $50 \mu \mathrm{g} / \mathrm{ml}$, respectively (data not shown). Compared with PHA, CD3/CD28 stimulation is more specific and more closely simulates the physiological conditions of the aforementioned CD147-related $\mathrm{T}$ cell stimulation signaling pathway, which could explain the difference between two groups. To further evaluate whether the inhibitory effects were mediated by the cytotoxicity of 5A12, peripheral CD4+ T cells were stained with Annexin V and PI. Even at the highest concentration $(1 \mathrm{mg} / \mathrm{ml})$, the addition of 5A12 did not significantly affect the rate of apoptosis (Annexin V+/PI- and Annexin V+/PI+) (Fig. 1C), indicating that the effects of 5A12 were not mediated by apoptosis.

$5 A 12$ inhibited the one-way MLR. The one-way MLR system is an effective method for evaluating an immunosuppressor in vitro. In the present study, we used it to analyze 

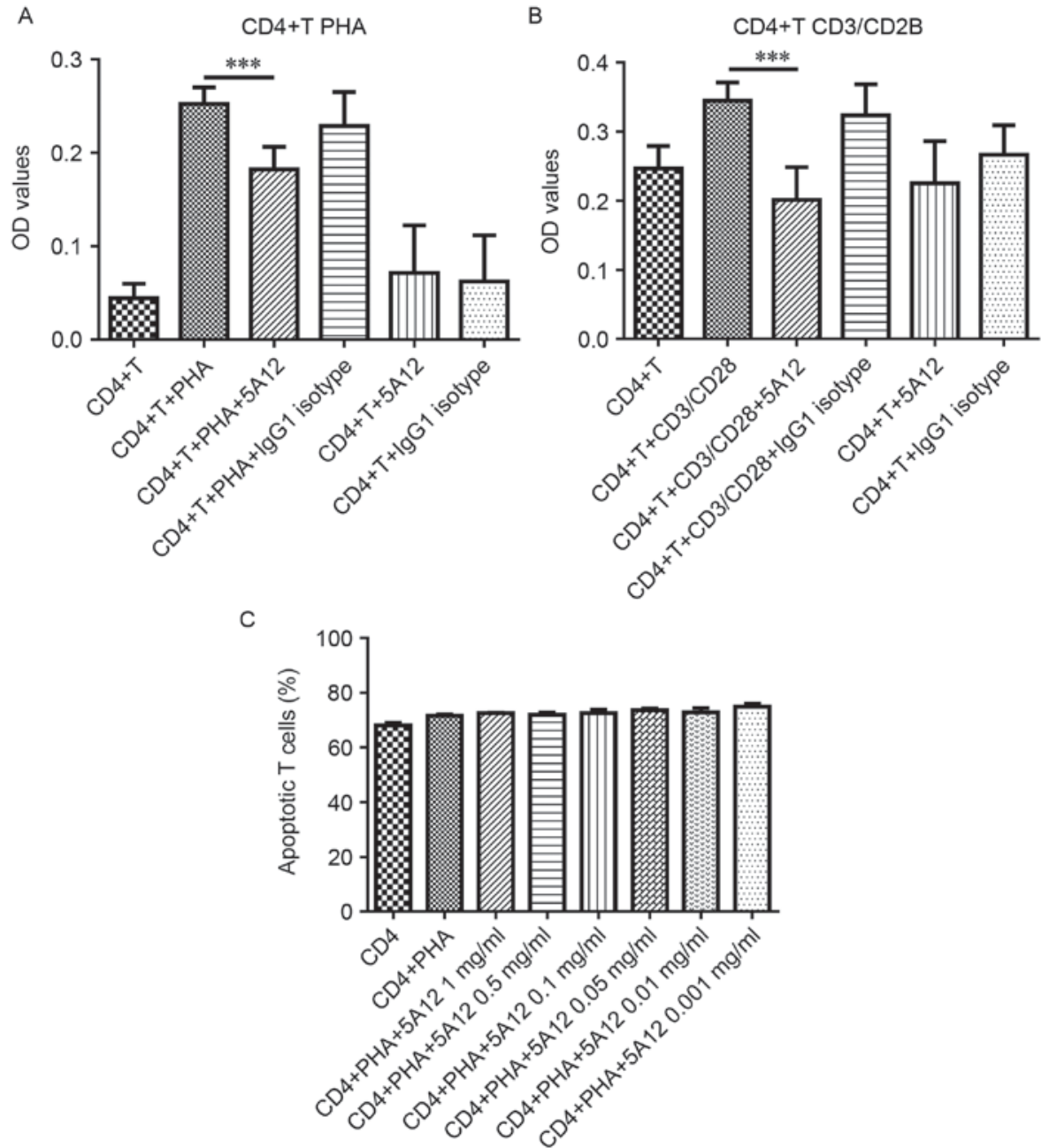

Figure 1.5A12 inhibited the proliferation of CD4+ T cells isolated from human peripheral blood without causing toxicity. Cells were stimulated with PHA (A) or CD3/CD28 magnetic beads (B) in the presence of 5A12 for $96 \mathrm{~h}$. The addition of 5A12 to the cultures strongly inhibited proliferation (A and B). CD4+ T cells were stimulated with PHA for $96 \mathrm{~h}$ in the presence of $5 \mathrm{~A} 12$ at $1,0.5,0.1,0.05,0.01$, or $0.001 \mathrm{mg} / \mathrm{ml}$, and the level of apoptosis was evaluated by flow cytometry after staining with FITC-conjugated Annexin V and PI (BioLegend) (C). Data are representative of three independent experiments. Multiple comparisons were performed with one-way ANOVA ${ }^{* * *} \mathrm{P}<0.001$.

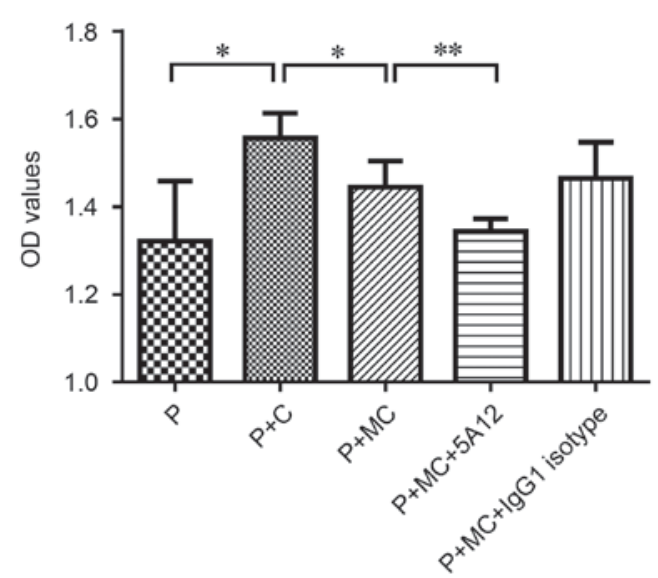

Figure 2. 5A12 inhibited the one-way MLR. PBMCs isolated from human volunteers served as responder cells, and splenocytes isolated from C57BL/6 mice served as stimulator cells. Cells were suspended in RPMI 1640 supplemented with $10 \%$ fetal bovine serum. The suspended cells were seeded at a density of $1.5 \times 10^{5}$ cells/well in round-bottomed 96 -well plates and cultured with or without 5A12 for $96 \mathrm{~h}$; then, lymphocyte proliferation was measured using a CCK- 8 assay. Data are representative of three independent experiments. Multiple comparisons were performed with one-way ANOVA ${ }^{*} \mathrm{P}<0.05,{ }^{* *} \mathrm{P}<0.01$. P: PBMCs, C: C57BL/6 mouse splenocytes, MC: Mitomycin-treated C57BL/6 mouse splenocytes. the immunosuppressive activity of 5A12; the protocol is included in the Materials and Methods section. When two MHC-mismatched lymphocytes are mixed, they will each promote the proliferation of the other. Lymphocytes incubated with mitomycin $\mathrm{C}$ lost their multiplication capacity and were used as stimulator cells. As shown in Fig. 2, 5A12 significantly inhibited the proliferation of the responder cells, i.e., the PBMCs, nearly to the point of equivalency with the baseline, unstimulated level. The minimum significant concentration was $200 \mu \mathrm{g} / \mathrm{ml}$ (data not shown). As the balance between Th cell subsets plays an important role in the development of graft rejection, we assessed the influence of 5A12 on expression of the Th1/Th2/Th17 cell-related cytokines IFN- $\gamma$, IL-4 and IL-17 from the culture supernatants. However, the addition of 5A12 had no influence on the expression of these cytokines.

Establishment of the allogeneic skin transplantation mouse model. To further clarify the immunosuppressive effects and underlying mechanisms, we assessed the in vivo effects of CD147 blockade using an allogeneic skin transplantation mouse model. Full-thickness skin grafts were obtained from the tails of $\mathrm{C} 57 \mathrm{BL} / 6\left(\mathrm{H}-2^{\mathrm{b}}\right)$ donor mice and transplanted onto 
A

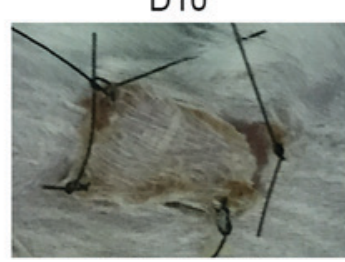

D13

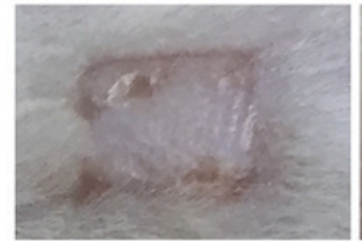

B

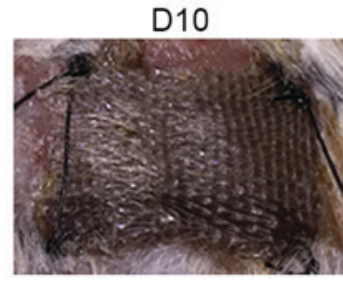

D13

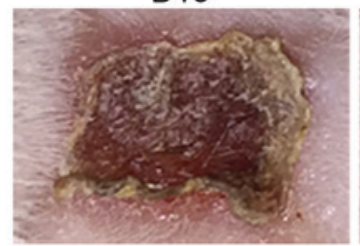

D11

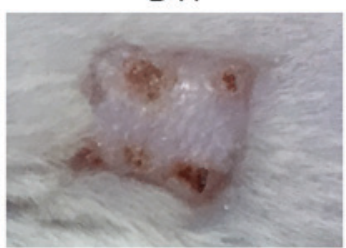

D14

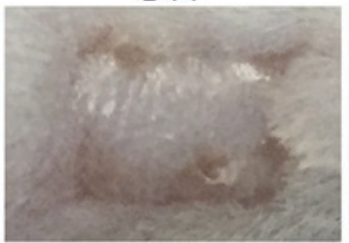

D11

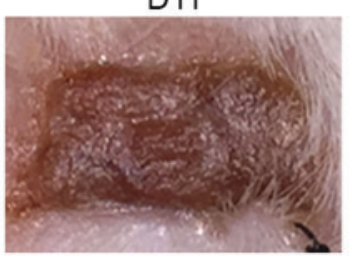

D14

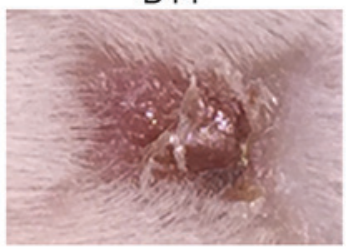

D12

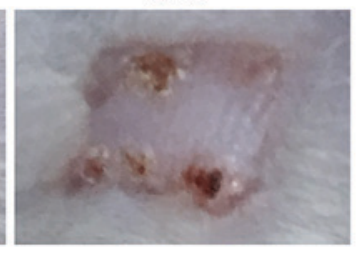

D30

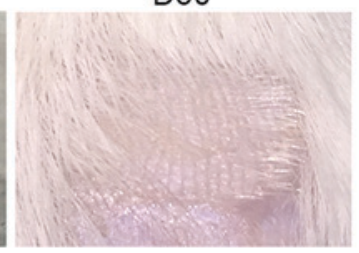

D12

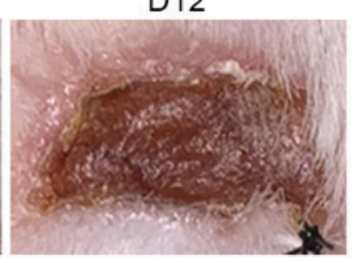

.

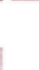

Figure 3. Establishment of the allogeneic skin transplantation model. Full-thickness skin grafts $1 \mathrm{~cm}$ in diameter were obtained from the tails of C57BL/6 $\left(\mathrm{H}-2^{\mathrm{b}}\right)$ donor mice and transplanted onto the backs of BALB/c $\left(\mathrm{H}-2^{\mathrm{d}}\right)$ recipient mice. On the tenth day after transplantation, the bandages were removed, and the skin grafts were observed and imaged every day thereafter. The images shown above were randomly chosen from the autologous transplantation (A) and allogeneic skin transplantation groups (B).

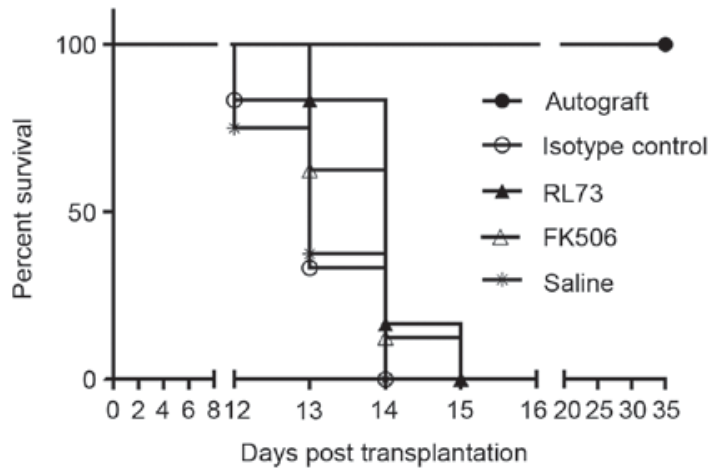

Figure 4. Blockade of CD147 with RL73 prolonged skin allograft survival. Skin allografts were transplanted from $\mathrm{C} 57 \mathrm{BL} / 6$ mice to BALB/c recipients, which were then intraperitoneally injected with RL73, its isotype control $(n=6)$, FK506 or equal volumes of saline $(n=8)$ every day from day 1 until complete rejection. Isogeneic skin transplantation was used as a control $(n=8)$. Graft survivals were analyzed by the Kaplan-Meier method using the log-rank test. RL73 and FK506 prolonged graft survival compared with that of the isotype control and saline groups (14 vs. 13 days; $\mathrm{P}<0.0001)$.

the backs of BALB/c $\left(\mathrm{H}-2^{\mathrm{d}}\right)$ recipient mice. On the tenth day after transplantation, the bandages were removed, and the skin grafts were observed every day. In the autologous transplantation group, the status of the skin grafts improved over time. On the 15th day after transplantation, the grafts began to grow new hair, indicating that the transplantation operation was successful (Fig. 3A). In contrast, grafts in the allogeneic skin transplantation group began to show signs of inflammation on the 10th day after transplantation. Due to rejection, the grafts turned red, and a yellow exudate was present around them. Over time, the skin tissue began to lose activity and become stiffer. By the 12th and 13th days, the skin grafts were either fully necrotic or had been rejected. Subsequently, scar tissue began to replace the wound (Fig. 3B).

CD147 blockade prolonged the survival of transplanted skin. We next assessed the in vivo effects of CD147 blockade using the allogeneic skin transplantation mouse model and a commercially functional anti-mouse CD147 antibody (RL73). To better evaluate the immunosuppressive effect of the antibody, we included a control group treated with a common effective immunosuppressant, Tacrolimus (FK506), which strongly inhibits $\mathrm{T}$ cell proliferation. From the day of the skin graft operation, these drugs were administered to the mice every day via intraperitoneal injection (RL73 and its isotype control, $5 \mathrm{mg} / \mathrm{kg}$; FK506, $0.5 \mathrm{mg} / \mathrm{kg}$ ). The mouse weight of each group did not differ, except for the FK506-treated mice, which showed slight weight loss compared with the other groups (data not shown). As shown in Fig. 4, mice that received RL73 and FK506 had a mean graft survival time of 
A

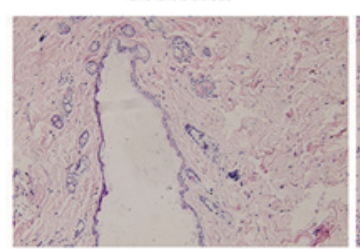

$\operatorname{RL} 73$

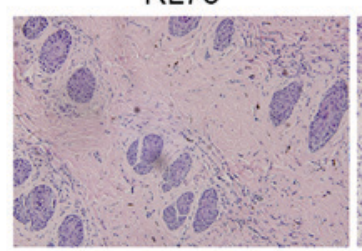

Autograft

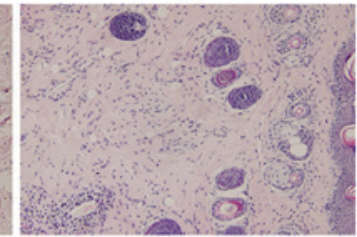

Isotype

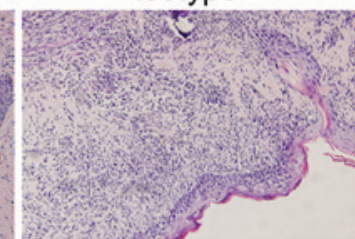

FK506

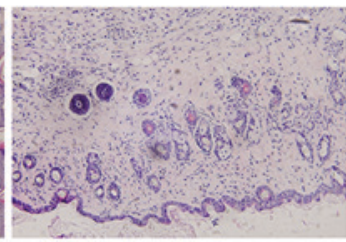

Saline

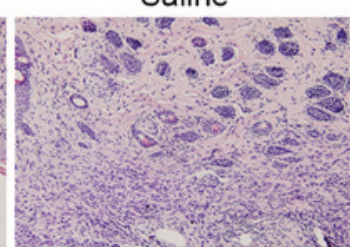

B

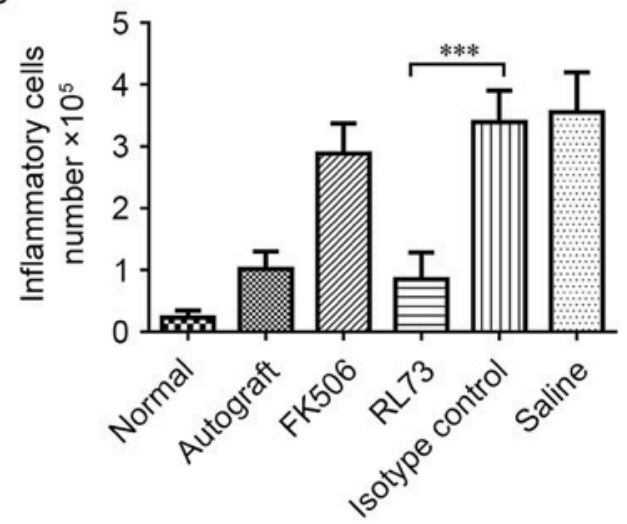

Figure 5. CD147 blockade decreased the level of inflammatory cell infiltration in transplanted skin. Skin allografts were transplanted from C57BL/6 mice to $B A L B / c$ recipients, which were then intraperitoneally injected with RL73, its isotype control ( $n=6$ ), FK506 or equal volumes of saline ( $n=8$ ) every day. Isogeneic skin transplantation was used as a control $(\mathrm{n}=8)$. Mice from each group were randomly selected and euthanized under general anesthesia on D10. Skin grafts were harvested and fixed in $4 \%$ formalin. Histological analysis of the skin grafts was performed by staining 5-mm sections of paraffin-embedded tissues with hematoxylin and eosin. Representative histological results are shown (A), magnification, x200. Inflammatory cells were counted by examining 10 different visual fields under a microscope at 400x magnification in a blinded manner (B). Multiple comparisons were performed with one-way ANOVA ${ }^{* * *} \mathrm{P}<0.001$.

14 days (range: $13-15$ days, $\mathrm{P}<0.05$ ) compared with salineand isotype control antibody-treated mice (range: 12-14 days). Although the mean survival time was prolonged by only one day, RL73 showed the same behavior as FK506 in skin transplantation.

CD147 blockade decreased the level of inflammatory cell infiltration in transplanted skin grafts. We then assessed the level of inflammatory infiltration in the skin grafts on the tenth day after transplantation; the rejection response is believed to be the strongest during this time. Hematoxylin-eosin staining showed there was little inflammatory infiltration in the normal skin and grafts of RL73-treated mice. Next, we counted the infiltrated inflammatory cells and found that in the RL73-treated group, the number was $75 \%$ lower than that in the isotype control-treated group. Similarly, the number of infiltrated inflammatory cells was 18\% lower in the FK506-treated mice than in the saline-treated mice, but this result was not significantly different $(\mathrm{P}=0.07)$ (Fig. 5A and $\mathrm{B})$.

CD147 blockade inhibited IL-17 expression in the serum of skin graft recipients. We also tested the serum levels of IFN- $\gamma$, IL-4 and IL-17 in skin graft recipients on the 10th day after transplantation. The serum expression levels of IL-4 and IFN- $\gamma$ were not significantly different among the groups, although the serum level of IL-4 was slightly increased in the RL72- and FK506-injected mice. However, the serum level of IL-17 was significantly decreased by RL73 to below the levels observed in normal mice (Fig. 6).

CD147 blockade decreased the proportions of CD4+ and $C D 8+$ memory $T$ cells in recipient mouse peripheral blood. To further elucidate the immunosuppressive effects of the CD147 antibodies and examine the altered IL-17 levels, on the 10th day after transplantation, the proportions of T cell subsets within the peripheral blood were detected in the skin graft recipients who received different treatments. We found that the proportions of CD4+ and CD8+ T cells were decreased by FK506 but not by RL73. Then, we assessed the proportions of Th1/Th2 and Th17/Treg cells. In the FK506- and RL73-treated groups, the levels of Th2 cells were slightly increased, but this result was not significant. As memory $\mathrm{T}$ cells are confirmed to be involved in development of the rejection reaction and to play an important role, we detected the proportions of both CD4+ and CD8+ memory T cells and found that they were sharply decreased (Fig. 7A and B). Compared with the isotype 
A

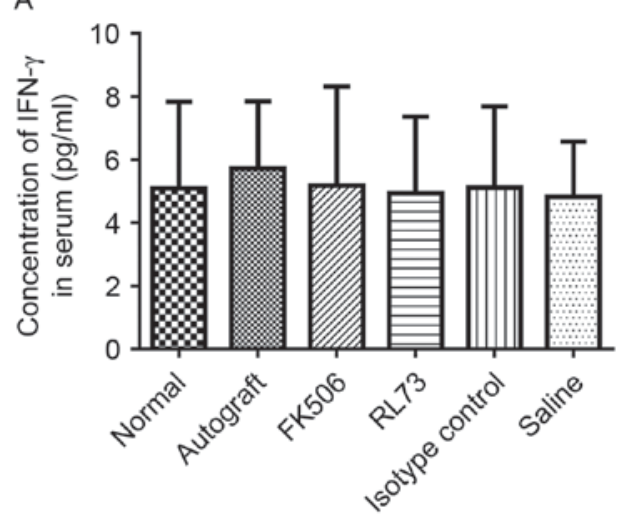

B

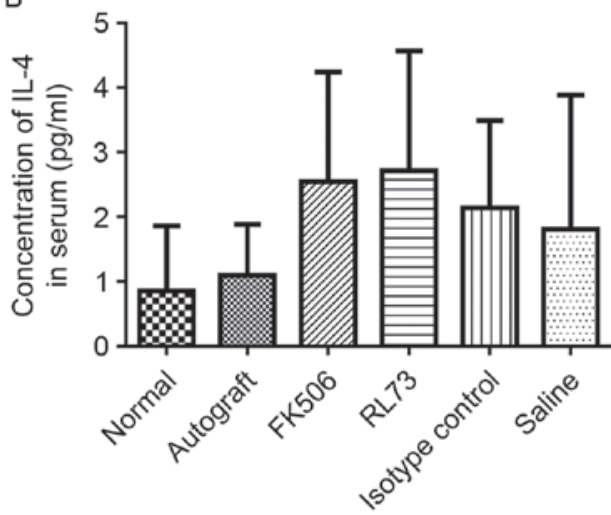

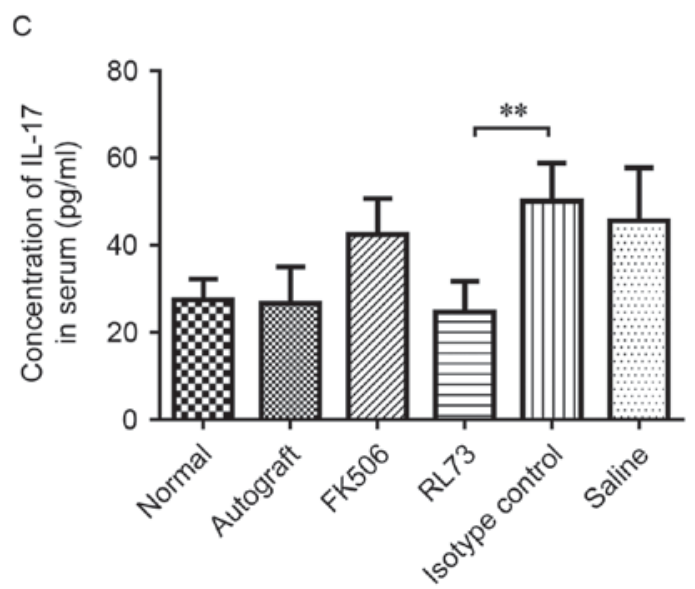

Figure 6. CD147 blockade inhibited serum IL-17 expression in skin graft recipients. Skin allografts were transplanted from C57BL/6 mice to BALB/c recipients, which were then intraperitoneally injected with RL73, the RL73 isotype control ( $n=6)$, FK506 or equal volumes of saline ( $n=8)$ every day. Mice from each group were randomly selected and euthanized under general anesthesia on D10 post-treatment. Blood was then collected from the orbital sinus. Plasma was obtained by centrifugation, and the serum levels of the cytokines IFN- $\gamma$ (A), IL-4 (B) and IL-17 (C) were measured using a MILLIPLEX MAP kit. Multiple comparisons were performed with one-way ANOVA ${ }^{* *} \mathrm{P}<0.01$.

control, the proportion of CD4+ memory $\mathrm{T}$ cells decreased to $70 \%$, and that of CD8+ memory $\mathrm{T}$ cells decreased to $80 \%$ (Fig. 7C and D). We also tested the proportions of the above $\mathrm{T}$ cell subsets within the spleen and found that the decreases in their proportions were not as significant as the decreases in their proportions in the peripheral blood; however, the proportion of CD4+ memory T cells in the spleen was still decreased compared with that in the peripheral blood.

\section{Discussion}

The specific inhibition of CD147 via blocking antibodies has been successfully tested in models of T cell-related diseases, including autoimmune diseases (e.g., RA (31-34) and multiple sclerosis (MS) $(35,36)$ and GVHD following bone marrow transplantation $(19,25,27,37)$. However, there have been no reports on this topic in the context of organ transplantation. Skin allograft rejection model is often characterized by acute rejection mediated by cellular immunity. It is widely used in basic pharmacodynamic studies or new methods for treating graft rejection reaction (38-40). Compared with other allograft models, such as heart or islet transplantation, the skin allograft rejection model has the advantage of being simple to create and easy to observe. On the other hand, the skin allograft rejection model is an aggressive model that is often resistant to immunosuppressive therapies. In our preliminary experiments, we used FK506, a clinically effective immunosuppressant, to score graft survival time and found that it was not particularly effective at the common doses used in clinical treatment, from 0.01 to $0.3 \mathrm{mg} / \mathrm{kg} / \mathrm{day}$. Then, higher doses of FK506, from 0.01-0.65 mg/kg/day, were tested. FK506 was ineffective at doses less than $0.5 \mathrm{mg} / \mathrm{kg} /$ day. Administering FK506 at a dose of $0.5 \mathrm{mg} / \mathrm{kg} /$ day prolonged the mean graft survival time by one additional day compared with doses less than $0.5 \mathrm{mg} / \mathrm{kg} / \mathrm{day}(\mathrm{P}<0.001)$; however, the mice began to show slight weight loss at this dose. At doses of more than $0.5 \mathrm{mg} / \mathrm{kg} / \mathrm{day}$, more obvious, serious side effects were observed. To elevate the efficacy of anti-CD147, we repeated the experiment in the same system.

In our study, the anti-mouse CD147 antibody exhibited the same effect as FK506 on prolonging skin graft survival and caused fewer side effects. In addition, the antibody mitigated inflammatory cell infiltration in transplanted skin and decreased the serum level of IL-17, a factor that plays an important role in initiation of the inflammatory response. IL-17 can promote the release of pro-inflammatory cytokines, thereby amplifying the inflammatory response (41). We found that the blockade of CD147 had no obvious influence on the proportions of Th17/Treg cells in the peripheral blood of recipient mice, although the proportion of $\mathrm{Th} 2$ cells showed 


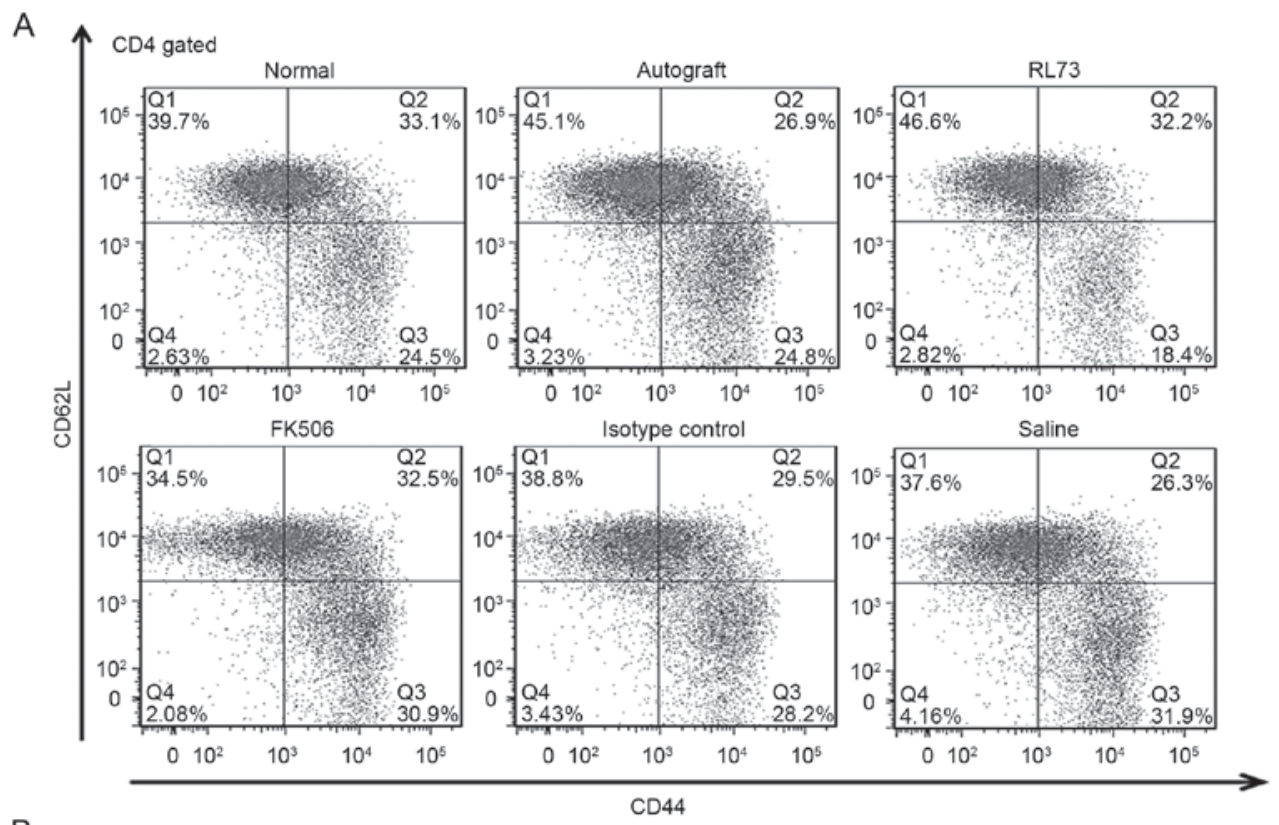

B

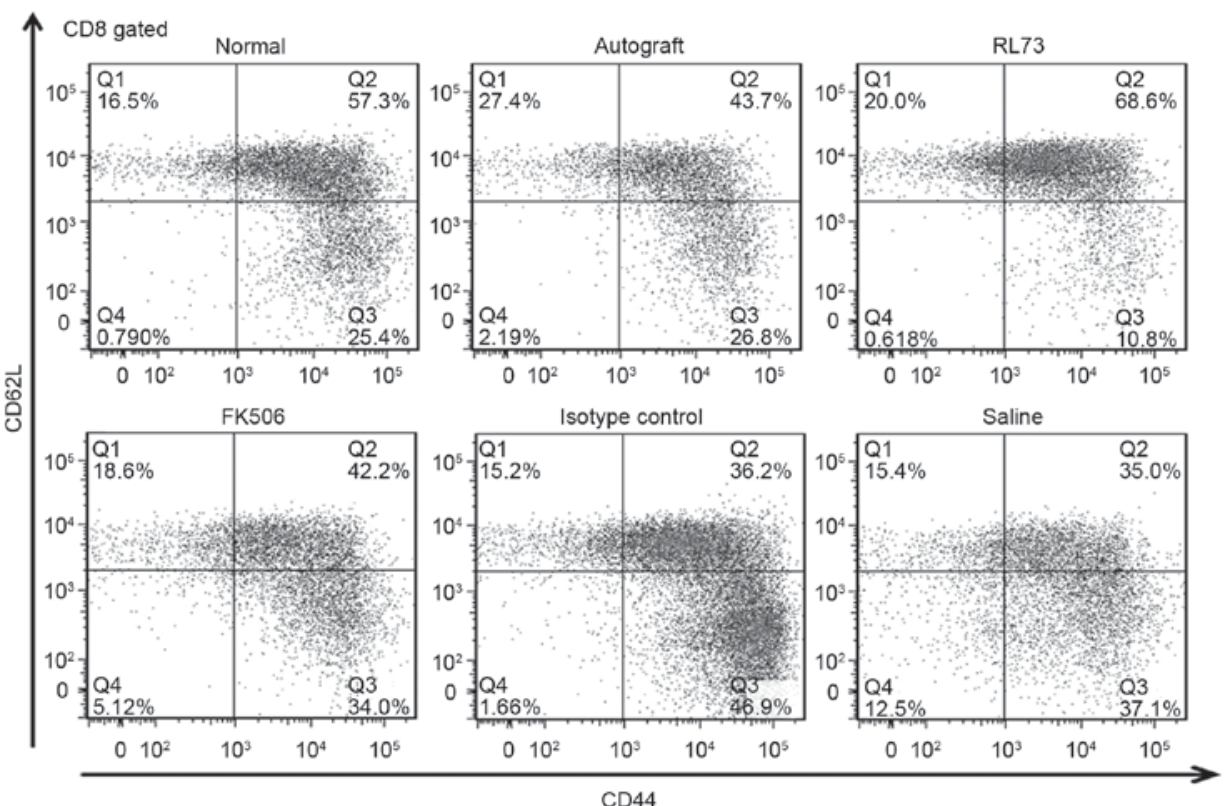

C

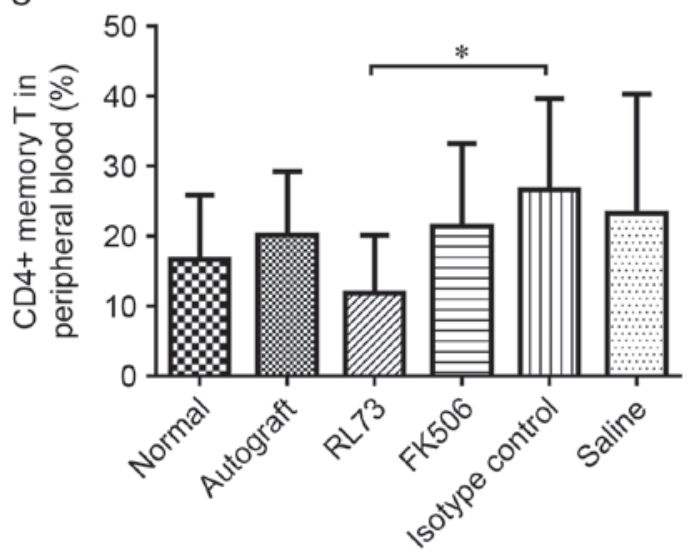

D

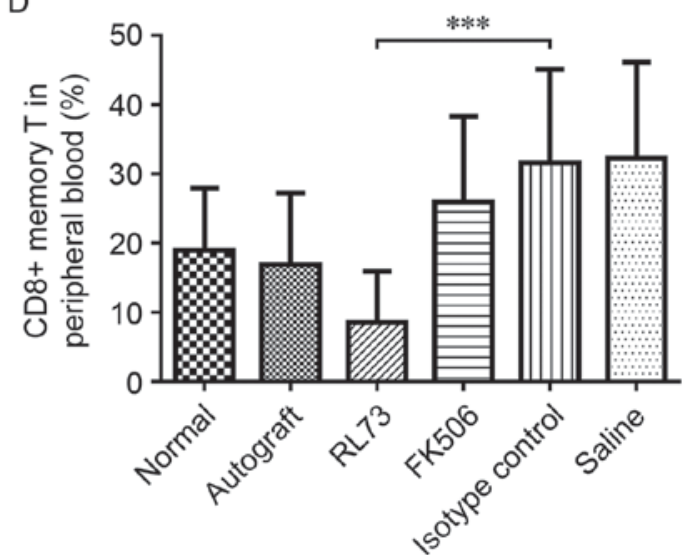

Figure 7. CD147 blockade decreased the proportions of CD4+ and CD8+ memory T cells within recipient peripheral blood. Skin allografts were transplanted from C57BL/6 mice to BALB/c recipients, which were then intraperitoneally injected with RL73, the RL73 isotype control (n=6), FK506 or equal volumes of saline ( $n=8)$ every day. Mice from each group were randomly selected and euthanized under general anesthesia on D10 post-treatment. Peripheral blood lymphocytes were stained with antibodies, according to conventional treatment methods, and then immediately detected using an LSRFortessa X-20 flow cytometer (BD Biosciences, USA). Representative flow cytometry results for the CD4+ memory T cells (CD4+ CD44 high CD62 L low) and CD8+ memory T cells (CD8+ CD44 high CD62 L low) are shown (A and B). The mean values of the CD4+ memory T cell (C) and CD8+ memory T cell proportions were calculated (D). Nonparametric Mann-Whitney U tests were performed ${ }^{*} \mathrm{P}<0.05,{ }^{* * *} \mathrm{P}<0.001$. 
a slight increase, which was consistent with the serum IL-4 expression level. However, these findings were insufficient to conclude that the blockade of CD147 changed the balance of Th1/Th2 cells. In contrast, the proportion of memory $\mathrm{T}$ cells (both CD4+ and CD8+ T cells) was dramatically decreased after CD147 blockade compared with those of the other groups. This effect may explain in part the decreased serum IL-17 level because some memory $\mathrm{T}$ cells can also secrete IL-17 (42). After transplantation, memory T cells quickly infiltrated the skin grafts and induced inflammation, leading to the infiltration and congregation of additional inflammatory cells. Compared with FK506-treated grafts, anti-CD147 treated grafts showed less lymphocyte infiltration. We speculate that memory $\mathrm{T}$ cell suppression may be the main mechanism underlying this phenomenon, as both drugs inhibit T cell activation, but only anti-CD147 treatment results in memory cell suppression. The majority of immunosuppressants currently used clinically exert their effects by blocking native lymphocyte homing and activation; however, memory $\mathrm{T}$ cells possess homing and activation behaviors that differ from those of native lymphocytes. These behaviors have rendered immunosuppressive drugs ineffective with respect to facilitating the inhibition of memory $\mathrm{T}$ cell-induced acute rejection. In addition, as mentioned above, memory $\mathrm{T}$ cell predominance is the most significant problem affecting graft tolerance after $\mathrm{T}$ cell depletion therapy. Perhaps agents that block CD147 are a potential solution to this problem.

In conclusion, the blockade of CD147 exerted an obvious immunosuppressive effect in a skin transplantation mouse model. This finding suggests that CD147 antibodies have potential for development into new target-specific immunosuppressant drugs. Furthermore, the humanized $\mathrm{mAb}$ against CD147 developed at our institute, i.e., 5A12, exerted a striking immunosuppressive effect in vitro. However, research on humanized $\mathrm{mAb}$ treatments is limited by the availability of suitable humanized animal models; work to overcome this difficulty is ongoing.

\section{Acknowledgements}

The present study was supported by the National Basic Research Program of China (973 Program), grant no. 2015CB553704. The authors thank Dr. Sheng Zhang from the Cell Engineering Research Center and Department of Cell Biology for her generous technical assistance and Dr. Tian Liu from the Department of Burns and Orthopedics at the First Affiliated Hospital of the Fourth Military Medical University for discussing the skin graft techniques and methods described herein.

\section{References}

1. Xie F, Chai J, Zhang Z, Hu Q and Ma T: MicroRNA 26a prolongs skin allograft survival and promotes regulatory $\mathrm{T}$ cell expansion in mice. Transpl Int 28: 1143-1151, 2015.

2. López MM, Valenzuela JE, Alvarez FC, López-Alvarez MR, Cecilia GS and Paricio PP: Long-term problems related to immunosuppression. Transpl Immunol 17: 31-35, 2006.

3. Ganschow R, Broering DC, Nolkemper D, Albani J, Kemper MJ, Rogiers X and Burdelski M: Th2 cytokine profile in infants predisposes to improved graft acceptance after liver transplantation. Transplantation 72: 929-934, 2001.
4. Gorczynski RM: Transplant tolerance modifying antibody to CD200 receptor, but not CD200, alters cytokine production profile from stimulated macrophages. Eur J Immunol 31: 2331-2337, 2001

5. Cousens LP, Orange JS and Biron CA: Endogenous IL-2 contributes to $\mathrm{T}$ cell expansion and IFN-gamma production during lymphocytic choriomeningitis virus infection. J Immunol 155: 5690-5699, 1995

6. Lu J and Zhang X: Immunological characteristics of renal transplant tolerance in humans. Mol Immunol 77: 71-78, 2016.

7. Gorbacheva V, Fan R, Li X and Valujskikh A: Interleukin-17 promotes early allograft inflammation. Am J Pathol 177: 1265-1273, 2010

8. Velasquez SY, Garcia LF, Opelz G, Alvarez CM and Süsal C: Release of soluble CD30 after allogeneic stimulation is mediated by memory $\mathrm{T}$ cells and regulated by IFN- $\gamma$ and IL- 2 . Transplantation 96: 154-161, 2013.

9. Donckier V, Craciun L, Miqueu P, Troisi RI, Lucidi V, Rogiers X, Boon N, Degré D, Buggenhout A, Moreno C, et al: Expansion of memory-type CD8+ T cells correlates with the failure of early immunosuppression withdrawal after cadaver liver transplantation using high-dose ATG induction and rapamycin. Transplantation 96: 306-315, 2013.

10. Gerlach UA, Vogt K, Schlickeiser S, Meisel C, Streitz M, Kunkel D, Appelt C, Ahrlich S, Lachmann N, Neuhaus P, et al: Elevation of CD4+ differentiated memory $\mathrm{T}$ cells is associated with acute cellular and antibody-mediated rejection after liver transplantation. Transplantation 95: 1512-1520, 2013.

11. Sallusto F, Lenig D, Förster R, Lipp M and Lanzavecchia A: Two subsets of memory $\mathrm{T}$ lymphocytes with distinct homing potentials and effector functions. Nature 401: 708-712, 1999.

12. Shan Z, Li Q, Zhou Y, Zeng X, Fan Q, Liao C, Zhu Y, Zhao Y, $\mathrm{Lu} \mathrm{X}$ and Liu J: Effect of CD4+ memory T cells on rejection response of ectopic heart transplantation in mice. Transplant Proc 43: 1989-1993, 2011.

13. Liang H, Liao C, Qi Z, Sha C, Xie B, Chen J, Xia J, Wang Y, Yao Q and Zhao Y: Rapamycin or tacrolimus alone fails to resist cardiac allograft accelerated rejection mediated by alloreactive CD4(+) memory T cells in mice. Transpl Immunol 22: 128-136, 2010.

14. Fearon DT, Manders P and Wagner SD: Arrested differentiation, the self-renewing memory lymphocyte, and vaccination. Science 293: 248-250, 2001.

15. Luckey CJ, Bhattacharya D, Goldrath AW, Weissman IL, Benoist $\mathrm{C}$ and Mathis D: Memory $\mathrm{T}$ and memory B cells share a transcriptional program of self-renewal with long-term hematopoietic stem cells. Proc Natl Acad Sci USA 103: 3304-3309, 2006.

16. Gurkan S, Luan Y, Dhillon N, Allam SR, Montague T, Bromberg JS, Ames S, Lerner S, Ebcioglu Z, Nair V, et al: Immune reconstitution following rabbit antithymocyte globulin. Am J Transplant 10: 2132-2141, 2010.

17. Sener A, Tang AL and Farber DL: Memory T-cell predominance following T-cell depletional therapy derives from homeostatic expansion of naive T cells. Am J Transplant 9: 2615-2623, 2009.

18. Engram JC, Cervasi B, Borghans JA, Klatt NR, Gordon SN Chahroudi A, Else JG, Mittler RS, Sodora DL, de Boer RJ, et al: Lineage-specific T-cell reconstitution following in vivo CD4+ and CD8+ lymphocyte depletion in nonhuman primates. Blood 116: 748-758, 2010.

19. Hahn JN, Kaushik DK and Yong VW: The role of EMMPRIN in T cell biology and immunological diseases. J Leukoc Biol 98: 33-48, 2015.

20. Yan L, Zucker S and Toole BP: Roles of the multifunctional glycoprotein, emmprin (basigin; CD147), in tumour progression. Thromb Haemost 93: 199-204, 2005.

21. Buck CA: Immunoglobulin superfamily: Structure, function and relationship to other receptor molecules. Semin Cell Biol 3: 179-188, 1992.

22. Williams AF and Barclay AN: The immunoglobulin superfamily-domains for cell surface recognition. Annu Rev Immunol 6: 381-405, 1988.

23. Kasinrerk W, Fiebiger E, Stefanová I, Baumruker T, Knapp W and Stockinger H: Human leukocyte activation antigen M6, a member of the Ig superfamily, is the species homologue of rat OX-47, mouse basigin and chicken HT7 molecule. J Immunol 149: 847-854, 1992.

24. Hu J, Dang N, Yao H, Li Y, Zhang H, Yang X, Xu J, Bian H, Xing J, Zhu P and Chen Z: Involvement of HAb18 G/CD147 in T cell activation and immunological synapse formation. J Cell Mol Med 14: 2132-2143, 2010. 
25. Deeg HJ, Blazar BR, Bolwell BJ, Long GD, Schuening F, Cunningham J, Rifkin RM, Abhyankar S, Briggs AD, Burt R, et al: Treatment of steroid-refractory acute graft-versus-host disease with anti-CD147 monoclonal antibody ABX-CBL. Blood 98: 2052-2058, 2001.

26. Bruner RJ and Farag SS: Monoclonal antibodies for the prevention and treatment of graft-versus-host disease. Semin Oncol 30: 509-519, 2003

27. Macmillan ML, Couriel D, Weisdorf DJ, Schwab G, Havrilla N Fleming TR, Huang S, Roskos L, Slavin S, Shadduck RK, et al: A phase $2 / 3$ multicenter randomized clinical trial of ABX-CBL versus ATG as secondary therapy for steroid-resistant acute graft-versus-host disease. Blood 109: 2657-2662, 2007.

28. Jeffery JR, Cheung K, Masniuk J and Taylor D: Mixed lymphocyte culture responses. Lack of correlation with cadaveric renal allograft survival and blood transfusions. Transplantation 38: 42-45, 1984.

29. Chong AS, Finnegan A, Jiang X, Gebel H, Sankary HN, Foster P and Williams JW: Leflunomide, a novel immunosuppressive agent. The mechanism of inhibition of $\mathrm{T}$ cell proliferation. Transplantation 55: 1361-1366, 1993.

30. Markees TG, Phillips NE, Gordon EJ, Noelle RJ, Shultz LD, Mordes JP, Greiner DL and Rossini AA: Long-term survival of skin allografts induced by donor splenocytes and anti-CD154 antibody in thymectomized mice requires CD4(+) T cells, interferon-gamma, and CTLA4. J Clin Invest 101: 2446-2455, 1998.

31. Wang CH, Dai JY, Wang L, Jia JF, Zheng ZH, Ding J, Chen ZN and Zhu P: Expression of CD147 (EMMPRIN) on neutrophils in rheumatoid arthritis enhances chemotaxis, matrix metalloproteinase production and invasiveness of synoviocytes. J Cell Mol Med 15: 850-860, 2011

32. Zhu P, Ding J, Zhou J, Dong WJ, Fan CM and Chen ZN Expression of CD147 on monocytes/macrophages in rheumatoid arthritis: Its potential role in monocyte accumulation and matrix metalloproteinase production. Arthritis Res Ther 7: R1023-R1033, 2005.

33. Jia J, Wang C, Shi Z, Zhao J, Jia Y, Zhao-Hui Z, Li X, Chen Z and Zhu P: Inhibitory effect of CD147/HAb18 monoclonal antibody on cartilage erosion and synovitis in the SCID mouse model for rheumatoid arthritis. Rheumatology (Oxford) 48: 721-726, 2009.
34. Damsker JM, Okwumabua I, Pushkarsky T, Arora K, Bukrinsky MI and Constant SL: Targeting the chemotactic function of CD147 reduces collagen-induced arthritis. Immunology 126: 55-62, 2009.

35. Agrawal SM, Silva C, Wang J, Tong JP and Yong VW: A novel anti-EMMPRIN function-blocking antibody reduces $\mathrm{T}$ cell proliferation and neurotoxicity: Relevance to multiple sclerosis. J Neuroinflammation 9: 64, 2012.

36. Agrawal SM, Silva C, Tourtellotte WW and Yong VW: EMMPRIN: A novel regulator of leukocyte transmigration into the CNS in multiple sclerosis and experimental autoimmune encephalomyelitis. J Neurosci 31: 669-677, 2011.

37. Bay JO, Cabrespine A and Peffault de Latour R: Role of monoclonal antibodies in the treatment of acute graft versus host disease. Bull Cancer 94: 33-41, 2007 (In French).

38. Mai HL, Boeffard F, Longis J, Danger R, Martinet B, Haspot F, Vanhove B, Brouard S and Soulillou JP: IL-7 receptor blockade following $\mathrm{T}$ cell depletion promotes long-term allograft survival. J Clin Invest 124: 1723-1733, 2014

39. Li J, Xiong T, Xiao R, Xiong A, Chen J, Altaf E, Zheng Y, Zhu G, He Y and Tan J: Anti-CCL25 antibody prolongs skin allograft survival by blocking CCR9 expression and impairing splenic T-cell function. Arch Immunol Ther Exp (Warsz) 61: 237-244, 2013.

40. Pasquet L, Douet JY, Sparwasser T, Romagnoli P and van Meerwijk JP: Long-term prevention of chronic allograft rejection by regulatory T-cell immunotherapy involves host Foxp3-expressing T cells. Blood 121: 4303-4310, 2013.

41. Diller ML, Kudchadkar RR, Delman KA, Lawson DH and Ford ML: Balancing Inflammation: The Link between Th17 and regulatory T cells. Mediators Inflamm 2016: 6309219, 2016.

42. Pappu R, Ramirez-Carrozzi V and Sambandam A: The interleukin-17 cytokine family: Critical players in host defence and inflammatory diseases. Immunology 134: 8-16, 2011. 